\title{
NONLINEAR MODEL PREDICTIVE CONTROL OF DYNAMIC POSITIONING OF DEEP-SEA SHIPS WITH A UNIFIED MODEL
}

\author{
Yuanhui Wang, ${ }^{*}$ Yulong Tuo, ${ }^{*}$ Simon X. Yang, ${ }^{* *}$ and Mingyu Fu*
}

\begin{abstract}
In this paper, a six degrees-of-freedom unified model of ship is established based on one dynamic positioning (DP) vessel, in which sea environment models of wind, current and waves are included. Then, the controller of DP ship based on a kind of nonlinear model predictive control algorithm is designed. By choosing an appropriate predictive period, the DP ship can move to the desired position and heading quickly and accurately. Finally, the stability of the developed control system for dynamic positioning of ships is proved by theoretical and simulation studies.
\end{abstract}

\section{Key Words}

Dynamic positioning, unified models, model predictive control, system stability

\section{Introduction}

Depending on the operational conditions, the vessel models may briefly be classified into stationkeeping, low-velocity and high-velocity models [1]. As shown in Sørensen [2], Fossen [3] and the references therein, different model reduction techniques are used for the various speed regimes. It will be difficult for the performance tests and design of controllers. In this context, a unified state-space model is presented in [4] which can contain different operational conditions and facilitate the design of controllers.

To maintain the position and heading of vessels, a system, called dynamic positioning (DP) system [5], was developed which can automatically compensate the environmental disturbance by means of active thrusters. Nowadays, offshore oil and gas industry in deep sea is the dominating market for DP vessels, as its cost does not increase with the increase in water depth and the operation. The overview of DP systems including references can be found

* College of Automation, Harbin Engineering University, Harbin, Heilongjiang Province, People's Republic of China; e-mail: \{wangyuanhui, tuoyulong, fumingyu\}@hrbeu.edu.cn

** Advanced Robotics and Intelligent Systems Laboratory, School of Engineering, University of Guelph, Guelph, ON, Canada; e-mail: syang@uoguelph.ca

Recommended by Prof. Chaomin Luo

(DOI: 10.2316/Journal.206.2016.6.206-4764) in Fay [6], Sørensen [2] and Sørensen et al. [7]. Many research groups worldwide have provided important results since the 1960s.

The DP system contains control system, position reference systems, sensors, the power system, thruster and propulsion system, independent joystick system and so on [5]. The DP control system is the key part among them. From a historical point of view, the proportional-integralderivative (PID) controller has been the dominating design technique since the 1960s. The PID control algorithm is a typical process control algorithm, which is simple and at low cost, but it may not be able to achieve the required control accuracy due to the limit of available control torque. In this context, many advanced modern control algorithms were presented, such as adaptive control [8], optimal control [9], intelligent control [10], model predictive control (MPC) [11] and so on. Among them, the adaptive control, optimal control and intelligent control are almost based on the intelligent algorithms. However, how to get the balance between real time and optimality is a big challenge for their applications. Under this circumstance, the MPC algorithm is widely used, whose effectiveness is due to the ease of its implementation (e.g. constraints on the control variables) and to the computation of optimal future sequences of manipulated variables for the maximization of performance [12].

Since the introduction of MPC presented by Richalet in 1978 [13], a number of relevant researches [14], [15], [16] were presented. Despite the success of linear MPC strategies, it is clear that their applicability to dynamic processes operating over wide operating regions would be strongly affected by the limited predictive capabilities of linear input-output empirical models [12].

Because of the shortage of the MPC algorithm, there has been an increasing focus on nonlinear model predictive control (NMPC) with rigorous dynamic models. NMPC technology has been widely used in aerospace, robotics and some other fields. Among the various NMPC algorithms, analytic MPC has its own advantages. The main features are that a specific analytical form of the optimal predictive controller is given, on-line optimization is unnecessary and the stability of closed-loop system is guaranteed [17]. Chen et al. presented a closed-form nonlinear predictive 


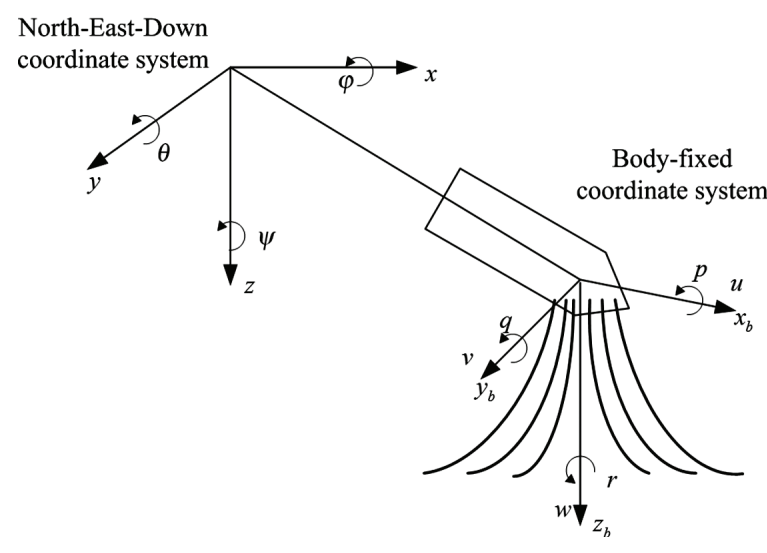

Figure 1. Reference coordinate system.

controller using approximation [18]. A fuzzy adaptive analytic MPC method is proposed by Peng et al. [19] for a class of uncertain nonlinear systems. But these control algorithms are just suitable for nonlinear systems with defined relative degree [20]. For the ill-defined relative degree nonlinear systems, Chen et al. presented a switching predictive control scheme to deal with it [21]. However, it is easy for the system to cause chattering. To avoid the chattering, Zhang et al. [20] brought forward a non-switch NMPC method for nonlinear systems with ill-defined relative degree. However, this algorithm has several strict conditions: defined robust relative degree for the system and available high-order derivative for the output. It is limited for this non-switch NMPC algorithm's application on the unified vessel mathematical model with ill-defined relative degree. Under these circumstances, the improved NMPC control algorithm is applied for the DP controller in this paper. There is no need to satisfy the strict conditions in $[20]$.

This paper is organized as follows: a six degrees-offreedoms (DOF) nonlinear unified model is established in Section 2 to describe the ship movement more accurately. An improved NMPC algorithm is exploited in Section 3 to the unified model of the DP ship in the harsh sea environment. The analysis of the stability is carried out in Section 4. Simulations are conducted to illustrate the performance of the proposed control algorithm in Section 5 . The conclusion remarks are drawn in section 6 , including some future work.

\section{Unified State-Space Model of Ships}

The positions and Euler angles of the ship are described by two rectangular coordinate systems: North-East-Down frame (n-frame) and Body-fixed frame (b-frame), as shown in Fig. 1, where $\eta=\left[\begin{array}{llllll}x & y & z & \phi & \theta & \psi\end{array}\right]^{T}$ and $\nu=\left[\begin{array}{llllll}u & v & w & p & q & r\end{array}\right]^{T}$ are the generalized positions and velocities.

According to Fossen [4], the unified mathematical model for DP ship is established which is given as follows:

$$
\begin{aligned}
& \dot{\eta}=J_{\Theta}\left(\Theta_{n b}\right) \nu \\
& M \dot{v}+D(\nu) v+\mu+G \eta=\tau_{e n v}+\tau
\end{aligned}
$$

where $M=M_{R B}+M_{A} \in R^{6 \times 6}, M_{R B}$ is the generalized rigid-body inertia matrix, $M_{A}$ is the added mass inertia matrix, $D(\nu)=D_{L}+D_{N L}(\nu) \in R^{6 \times 6}, D_{L}$ is the linear damping matrix and $D_{N L}(\nu)$ is the nonlinear damping matrix. The restoring forces $g(\eta)$ can be linearized into $G \eta$ according to Faltinsen and Svensen [22], $\tau_{e n v}$ is a vector of environmental forces due to waves and wind and $\tau$ is the generalized control forces. Because DP ship's speed is very low, Coriolis and centripetal matrices are ignored. Matrix $J_{\Theta}\left(\Theta_{n b}\right)$ is the kinematic transformation matrix given by the following equation:

$$
\begin{gathered}
J_{\Theta}\left(\Theta_{n b}\right)=\left[\begin{array}{cc}
R_{b}^{n}\left(\Theta_{n b}\right) & 0_{3 \times 3} \\
0_{3 \times 3} & T_{\Theta}\left(\Theta_{n b}\right)
\end{array}\right] \\
R_{b}^{n}\left(\Theta_{n b}\right)=\left(\begin{array}{ccc}
c \psi c \theta & -s \psi c \varphi+c \psi s \theta s \varphi & s \psi s \varphi+c \psi c \varphi s \theta \\
s \psi c \theta & c \psi c \varphi+s \psi s \theta s \varphi & -c \psi s \varphi+s \theta s \psi c \varphi \\
-s \theta & c \theta s \varphi & c \theta c \varphi
\end{array}\right) \\
T_{\Theta}\left(\Theta_{n b}\right)=\left(\begin{array}{ccc}
1 & s \varphi t \theta & c \varphi t \theta \\
0 & c \varphi & -s \varphi \\
0 & s \varphi / c \theta & c \varphi / c \theta
\end{array}\right)
\end{gathered}
$$

where $s \cdot=\sin (\cdot), c \cdot=\cos (\cdot)$ and $t \cdot=\tan (\cdot)$.

Then, $\mu$ is the memory effect of the fluid [3]. It can be calculated as follows:

$$
\mu=\int_{-\infty}^{t} K(t-\tau) \delta \nu(\tau) d \tau=\int_{0}^{t} K(t-\tau) \delta \nu_{r}(\tau) d \tau
$$

where $K(t-\tau)$ is the retardation function, $\delta \nu(t)=\nu(t)-$ $\bar{\nu}(t)$ and $\bar{\nu}(t)=\left[\begin{array}{llllll}U & 0 & 0 & 0 & 0 & 0\end{array}\right]^{T}$. If $\delta \nu$ is a unit impulse, (5) can be approximated as a linear state-space model [23]:

$$
\left\{\begin{array}{l}
\dot{\chi}=A_{r} \chi+B_{r} \delta v, \\
\mu=C_{r} \chi+D_{r} \delta v,
\end{array} \quad \chi(0)=0\right.
$$

where $A_{r}, B_{r}, C_{r}$ and $D_{r}$ are constant matrices.

At last, the effects of ocean current disturbance can be considered in the system as follows:

$$
\begin{aligned}
v_{r} & =\left[\begin{array}{lllllll}
u-u_{c} & v-v_{c} & w & p & q & r
\end{array}\right]^{T} \\
& =\left[\begin{array}{llllll}
u_{r c} & v_{r c} & w & p & q & r
\end{array}\right]^{T}
\end{aligned}
$$

where $u_{c}$ and $v_{c}$ are the velocity components of current. So, (1) can be rewritten as follows:

$$
\begin{aligned}
& \dot{\eta}=J_{\Theta}\left(\Theta_{n b}\right) \nu \\
& M \dot{v}_{r}+D\left(v_{r}\right) v_{r}+\mu+G \eta=\tau_{e n v}+\tau
\end{aligned}
$$




\section{Controller Design}

For simplicity without the loss of generality, only three degrees of freedom (surge, sway and yaw) are considered in (8) as follows:

$$
\begin{aligned}
& \dot{\eta}=R(\psi) \nu \\
& M \dot{v}+D v=\tau_{\text {env }}+\tau-\mu=\tau_{\text {total }}
\end{aligned}
$$

Then, $M, D$ and the kinematic transformation matrix $R(\psi)$ will take the following forms:

$$
\begin{aligned}
R(\psi) & =\left[\begin{array}{ccc}
\cos \psi & -\sin \psi & 0 \\
\sin \psi & \cos \psi & 0 \\
0 & 0 & 1
\end{array}\right] \\
M & =\left[\begin{array}{ccc}
m_{11} & 0 & 0 \\
0 & m_{22} & m_{23} \\
0 & m_{32} & m_{33}
\end{array}\right] \\
D & =\left[\begin{array}{ccc}
d_{11} & 0 & 0 \\
0 & d_{22} & d_{23} \\
0 & d_{32} & d_{33}
\end{array}\right]
\end{aligned}
$$
follows:

According to the literature [4], $\mu$ can be expressed as

$$
\mu=\left[\begin{array}{l}
\mu_{X} \\
\mu_{Y} \\
\mu_{N}
\end{array}\right]=\left[\begin{array}{c}
\mu_{X} \\
\mu_{Y 1}+\mu_{Y 2} \\
\mu_{N 1}+\mu_{N 2}
\end{array}\right]
$$

where $\mu_{X}$ can be calculated as the following equation:

$$
\begin{aligned}
& \dot{\xi}_{X}=A_{X} \xi_{X}+B_{X} u \\
& \mu_{X}=C_{X} \xi_{X}+D_{X} u
\end{aligned}
$$

where $A_{X} \in R_{5 \times 5}, B_{X} \in R_{5 \times 1}, C_{X} \in R_{1 \times 5}, D_{X} \in R_{1 \times 1}$ and $\xi_{X} \in R_{5 \times 1}$ are coefficient matrices. The remaining four variables are also available with the same method.

Then, (9) can be expanded to differential equations as follows:

$$
\left\{\begin{array}{l}
\dot{x}=u \cos \psi-v \sin \psi \\
\dot{y}=u \sin \psi+v \cos \psi \\
\dot{\psi}=r \\
\dot{u}=-\frac{d_{11}}{m_{11}} u+\frac{1}{m_{11}} \tau_{\text {total } X} \\
\dot{v}=-\frac{d_{22}}{m_{22}} v-\frac{d_{23}}{m_{22}} r+\frac{1}{m_{22}} \tau_{\text {totalY }} \\
\dot{r}=-\frac{d_{32}}{m_{33}} v-\frac{d_{33}}{m_{33}} r+\frac{1}{m_{33}} \tau_{\text {totalN }} \\
y_{1}=x \\
y_{2}=y \\
y_{3}=\psi
\end{array}\right.
$$

where $y_{1}, y_{2}$ and $y_{3}$ are outputs of the system.

Equation (15) can be rewritten as follows:

$$
\begin{aligned}
\dot{\boldsymbol{x}} & =f(\boldsymbol{x})+g(\boldsymbol{x}) \boldsymbol{u} \\
\boldsymbol{y} & =h(\boldsymbol{x})
\end{aligned}
$$

where

$$
\begin{aligned}
\boldsymbol{x} & =\left[\begin{array}{llllll}
x_{1} & x_{2} & x_{3} & x_{4} & x_{5} & x_{6}
\end{array}\right]^{T} \\
& =\left[\begin{array}{llllll}
x & y & \psi & u & v & r
\end{array}\right]^{T} \\
\boldsymbol{u} & =\left[\begin{array}{llll}
u_{1} & u_{2} & u_{3}
\end{array}\right]^{T} \\
& =\left[\begin{array}{llll}
\tau_{\text {totalX }} & \tau_{\text {totalY }} & \tau_{\text {totalN }}
\end{array}\right]^{T}
\end{aligned}
$$

$$
\begin{aligned}
\boldsymbol{y} & =\left[\begin{array}{lll}
y_{1} & y_{2} & y_{3}
\end{array}\right]^{T} \\
& =\left[\begin{array}{lll}
h_{1}(x) & h_{2}(x) & h_{3}(x)
\end{array}\right]^{T}=\left[\begin{array}{lll}
x & y & \psi
\end{array}\right]^{T}
\end{aligned}
$$

The NMPC algorithm should be valued by a suitable performance index. Thus, a relatively accurate and comprehensive predictive control performance [24] can be approximated as follows:

$$
\begin{aligned}
J= & \frac{1}{2} \lambda_{1}\left[\hat{\boldsymbol{y}}(t+T)-\hat{\boldsymbol{y}}_{d}(t+T)\right]^{T}\left[\hat{\boldsymbol{y}}(t+T)-\hat{\boldsymbol{y}}_{d}(t+T)\right] \\
& +\frac{1}{2} \int_{0}^{T}\left[\lambda_{2}\left(\hat{\boldsymbol{y}}(t+T)-\hat{\boldsymbol{y}}_{d}(t+T)\right)^{T}(\hat{\boldsymbol{y}}(t+T)\right. \\
& \left.\left.-\hat{\boldsymbol{y}}_{d}(t+T)\right)\right] d \zeta \\
& +\frac{1}{2} \int_{0}^{T}\left[\lambda_{3} \hat{\boldsymbol{u}}^{T}(t+\zeta) \hat{\boldsymbol{u}}(t+\zeta)\right] d \zeta \\
= & \frac{1}{2} \lambda_{1} \hat{\boldsymbol{e}}(t+T)^{T} \hat{\boldsymbol{e}}(t+T) \\
& +\frac{1}{2} \int_{0}^{T}\left[\lambda_{2} \hat{\boldsymbol{e}}(t+T)^{T} \hat{\boldsymbol{e}}(t+T)+\lambda_{3} \hat{\boldsymbol{u}}^{T}(t+\zeta) \hat{\boldsymbol{u}}(t+\zeta)\right] d \zeta
\end{aligned}
$$


where $T$ is the predictive period and the tracking error defined as the following form: $\hat{\boldsymbol{e}}(t+\zeta)=\hat{\boldsymbol{y}}(t+\zeta)-\hat{\boldsymbol{y}}_{d}(t+\zeta)$, $\hat{\boldsymbol{y}}(t+\zeta)$ and $\hat{\boldsymbol{y}}_{d}(t+\zeta)$ stand for the predicted output and the desired predicted output. $\lambda_{1}, \lambda_{3} \geq 0$ and $\lambda_{2}>0$ reflect the proportions of the terminal constraint of the output, the error of the output and the control sequence, respectively. The superior character " $\wedge$ " represents that the variable is predicted and $\hat{\boldsymbol{y}}_{d}(t+\zeta)$ is usually constant.

In a receding cycle $[t, t+T]$, the dynamic equation for (2) can be written as follows:

$$
\left\{\begin{array}{l}
\dot{\hat{\boldsymbol{x}}}(t+\zeta)=f(\hat{\boldsymbol{x}}(t+\zeta))+g(\hat{\boldsymbol{x}}(t+\zeta)) \hat{\boldsymbol{u}}(t+\zeta), \\
\hat{\boldsymbol{y}}(t+\zeta)=h(\hat{\boldsymbol{x}}(t+\zeta)),
\end{array} \quad \zeta \in[0, T]\right.
$$

where $\hat{\boldsymbol{x}}(t+\zeta)$ and $\hat{\boldsymbol{u}}(t+\zeta)$ are state and input, respectively. The initial value of $\hat{\boldsymbol{x}}(t+\zeta)$ in a receding cycle should meet the following form:

$$
\hat{\boldsymbol{x}}(t+\zeta)=\hat{\boldsymbol{x}}(t), \quad \zeta=0
$$

According to (21) and (22), if the output makes the performance minimal, the optimal input of the controller will be achieved. Actually, the initial value of the optimal control law can be used in the system to meet the requirements:

$$
\boldsymbol{u}(t)=\hat{\boldsymbol{u}}^{*}(t+\zeta), \quad \zeta=0
$$

Receding optimization and feedback correction are in progress at every time of $t$ and the performance index $J$ becomes smaller and smaller so that the actual output keeps approaching the desired output.

Consider practical engineering applications, the assumption about the input is as follows:

$$
\hat{\boldsymbol{u}}(t+\zeta)=\boldsymbol{u}(t)=\mathrm{const}, \quad \zeta \in[0, T]
$$

Before the design of controller, $\hat{\boldsymbol{y}}(t+\zeta)$ and $\hat{\boldsymbol{y}}_{d}(t+\zeta)$ can be rewritten as follows:

$$
\begin{gathered}
\hat{\boldsymbol{y}}(t+\zeta)=\left[\begin{array}{l}
\hat{y}_{1}(t+\zeta) \\
\hat{y}_{2}(t+\zeta) \\
\hat{y}_{3}(t+\zeta)
\end{array}\right]=\left[\begin{array}{l}
\hat{x}(t+\zeta) \\
\hat{y}(t+\zeta) \\
\hat{\psi}(t+\zeta)
\end{array}\right] \\
\hat{\boldsymbol{y}}_{d}(t+\zeta)=\left[\begin{array}{l}
\hat{y}_{1 d}(t+\zeta) \\
\hat{y}_{2 d}(t+\zeta) \\
\hat{y}_{3 d}(t+\zeta)
\end{array}\right]=\left[\begin{array}{l}
\hat{x}_{d}(t+\zeta) \\
\hat{y}_{d}(t+\zeta) \\
\hat{\psi}_{d}(t+\zeta)
\end{array}\right]
\end{gathered}
$$

The predicted output and the predicted desired output will be approximated by its Taylor-series expansion to any specified accuracy as follows:

$$
\begin{gathered}
\hat{\boldsymbol{y}}(t+\zeta)=\boldsymbol{\xi}^{T}(\zeta) \chi(t) \\
\hat{\boldsymbol{y}}_{d}(t+\zeta)=\boldsymbol{\xi}^{T}(\zeta) \chi_{d}(t)
\end{gathered}
$$

where

$$
\begin{aligned}
& \boldsymbol{\xi}(\zeta)=\left[\begin{array}{lll}
\boldsymbol{\xi}_{1} & & \\
& \boldsymbol{\xi}_{2} & \\
& & \boldsymbol{\xi}_{3}
\end{array}\right] \\
& \boldsymbol{\xi}_{1}=\boldsymbol{\xi}_{2}=\boldsymbol{\xi}_{3}=\left[\begin{array}{lllll}
1 & \zeta & \frac{\zeta^{2}}{2 !} & \cdots & \frac{\zeta^{N}}{N !}
\end{array}\right]^{T} \\
& \chi(t)=\left[\begin{array}{lll}
\chi_{1}^{T}(t) & \chi_{2}^{T}(t) & \chi_{3}^{T}(t)
\end{array}\right]^{T}
\end{aligned}
$$

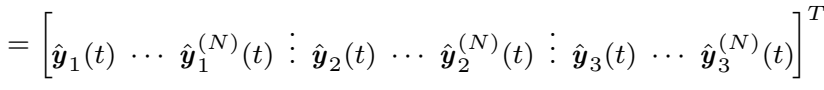

$$
\begin{aligned}
& \chi_{d}(t)=\left[\begin{array}{lll}
\chi_{1 d}^{T}(t) & \chi_{2 d}^{T}(t) & \chi_{3 d}^{T}(t)
\end{array}\right]^{T}
\end{aligned}
$$

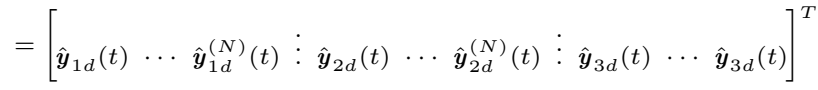

To simplify, $N$ is defined as 3 . Then invoking (27) into (20), the approximate expansion of $J$ is as follows:

$$
J \approx \frac{1}{2}\left[\chi(t)-\chi_{d}(t)\right]^{T} M\left[\chi(t)-\chi_{d}(t)\right]+\frac{1}{2} \lambda_{3} T \hat{\boldsymbol{u}}^{T} \hat{\boldsymbol{u}}
$$

where

$$
\begin{aligned}
& M=\left.\lambda_{1} \boldsymbol{\xi}(\zeta) \boldsymbol{\xi}^{T}(\zeta)\right|_{\zeta=T}+\lambda_{2} \int_{0}^{T} \boldsymbol{\xi}(\zeta) \boldsymbol{\xi}^{T}(\zeta) d \zeta \\
& =\left[\begin{array}{lll}
M^{(1)} & & \\
& & \\
& M^{(2)} & \\
& & M^{(3)}
\end{array}\right]
\end{aligned}
$$

If following (34) is satisfied, then $J$ will be minimal:

$$
\begin{aligned}
& \left(\frac{\partial \chi(t)}{\partial \hat{\boldsymbol{u}}}\right)^{T} M\left(\chi(t)-\chi_{d}(t)\right)+\lambda_{3} T \hat{\boldsymbol{u}}=0 \\
& \left(\frac{\partial \chi(t)}{\partial \hat{\boldsymbol{u}}}\right)^{T} M \frac{\partial \chi(t)}{\partial \hat{\boldsymbol{u}}}+\left(\frac{\partial \chi(t)}{\partial \hat{\boldsymbol{u}}}\right)^{T} M\left(\chi(t)-\chi_{d}(t)\right)+\lambda_{3} T>0
\end{aligned}
$$

According to $(24), \hat{\boldsymbol{u}}^{(i)}=\mathbf{0}(i \geq 1)$ is easily obtained. So, $\hat{y}_{j}^{(i)}(t),(i \geq 1)$ which conclude $\hat{\boldsymbol{u}}^{(i)}(i \geq 1)$ can be set to zero. For simplicity, the following notation is introduced as follows:

$$
\hat{\boldsymbol{u}}^{2}=\left[\begin{array}{llllll}
\hat{u}_{1}^{2} & \hat{u}_{1} \hat{u}_{2} & \hat{u}_{2}^{2} & \hat{u}_{2} \hat{u}_{3} & \hat{u}_{3}^{2} & \hat{u}_{3} \hat{u}_{1}
\end{array}\right]^{T}
$$

Before calculating $\hat{y}_{j}^{(i)}(t),(i \geq 0)$, a mathematical toolLie derivative [25] need to be introduced. Below is its concept.

Definition 1. Given a function $h(x): x \rightarrow R$ and a vector field $f(x)=\left[f_{1} \ldots f_{n}\right]^{T}$ defined on $x=\left[x_{1} \ldots x_{n}\right]^{T}$, the Lie 
derivative can be interpreted as the directional derivative of $h$ along $f$ :

$$
L_{f} h=\nabla h \cdot f(x)=\frac{\partial h}{\partial x} \cdot f(x)
$$

The high-order Lie derivative is defined as follows:

$$
L_{f}^{i} h=L_{f}\left(L_{f}^{i-1} h\right)=\nabla\left(L_{f}^{i-1} h\right) \cdot f(x), \quad \forall i=1,2, \ldots
$$

Specially, the zero-order Lie derivative is defined as follows:

$$
L_{f}^{0} h=h
$$

If $g$ is another vector field defined on $x$, define that:

$$
L_{g} L_{f} h=\nabla\left(L_{f} h\right) \cdot g=\frac{\partial L_{f} h}{\partial x} \cdot g(x)
$$

The form of $\hat{y}_{k}(t)$ and its derivatives are as follows:

$$
\begin{aligned}
& \hat{y}_{k}(t)=q k_{0,0} \\
& \hat{y}_{k}^{(1)}(t)=q k_{1,0} \\
& \hat{y}_{k}^{(2)}(t)=q k_{2,0}+q k_{2,1} \hat{\boldsymbol{u}} \\
& \hat{y}_{k}^{(3)}(t)=q k_{3,0}+q k_{3,1} \hat{\boldsymbol{u}}+q k_{3,2} \hat{\boldsymbol{u}}^{2}
\end{aligned}
$$

where

$$
\begin{gathered}
q k_{0,0}=h_{k} \\
q k_{1,0}=L_{f} h_{k} \\
q k_{2,0}=L_{f}^{2} h_{k} \\
q k_{2,1}=\left[\begin{array}{lll}
L_{g_{1}} L_{f} h_{k} & L_{g_{2}} L_{f} h_{k} & L_{g_{3}} L_{f} h_{k}
\end{array}\right] \\
q k_{3,0}=L_{f}^{3} h_{k} \\
q k_{3,1}=\left[\begin{array}{c}
L_{g_{1}} L_{f}^{2} h_{k}+L_{f} L_{g_{1}} L_{f} h_{k} \\
L_{g_{2}} L_{f}^{2} h_{k}+L_{f} L_{g_{2}} L_{f} h_{k} \\
L_{g_{3}} L_{f}^{2} h_{k}+L_{f} L_{g_{3}} L_{f} h_{k}
\end{array}\right]^{T} \\
q k_{3,2}=\left[\begin{array}{c}
L_{g_{1}}^{2} L_{f} h_{k} \\
L_{g_{2}} L_{g_{1}} L_{f} h_{k}+L_{g_{1}} L_{g_{2}} L_{f} h_{k} \\
L_{g_{2}}^{2} L_{f} h_{k} \\
L_{g_{3}} L_{g_{2}} L_{f} h_{k}+L_{g_{2}} L_{g_{3}} L_{f} h_{k} \\
L_{g_{3}}^{2} L_{f} h_{k} \\
L_{g_{3}} L_{g_{1}} L_{f} h_{k}+L_{g_{1}} L_{g_{3}} L_{f} h_{k}
\end{array}\right] k=1,2,3
\end{gathered}
$$

According to the definition of Lie derivative, several calculation results can be obtained as follows:

$$
\begin{aligned}
& L_{g_{1}} L_{f} h_{1}=1 / m_{11} \cos x_{3} \\
& L_{g_{2}} L_{f} h_{1}=-1 / m_{22} \sin x_{3} \\
& L_{g_{3}} L_{f}^{2} h_{1}=1 / m_{33}\left(-x_{4} \sin x_{3}-x_{5} \cos x_{3}+d_{23 / m_{22}} \sin x_{3}\right) \\
& L_{g_{1}} L_{f} h_{2}=1 / m_{11} \sin x_{3} \\
& L_{g_{2}} L_{f} h_{2}=1 / m_{22} \cos x_{3} \\
& L_{g_{3}} L_{f}^{2} h_{2}=1 / m_{33}\left(x_{4} \cos x_{3}-x_{5} \sin x_{3}-d_{23} / m_{22} \cos x_{3}\right)
\end{aligned}
$$

where $r_{i j}$ is the relative degree between the $i$ th input and the $j$ th output.

It is possible for the system state $x$ to be zero. So, $r_{11}$, $r_{21}, r_{31}, r_{12}, r_{22}$ and $r_{32}$ are ill-defined relative degrees. It can be seen that the mathematical model of DP vessel is a nonlinear system with ill-defined relative degree.

For simplicity, the following notation is introduced:

$$
q_{., i}(\boldsymbol{x})=\left[\begin{array}{l}
q 1_{, i}(\boldsymbol{x}) \\
q 2_{, i}(\boldsymbol{x}) \\
q 3_{, i}(\boldsymbol{x})
\end{array}\right], \quad i=0,1,2
$$

where

$$
\begin{gathered}
q k_{., i}(\boldsymbol{x})=\left[\begin{array}{c}
q k_{0, i}(\boldsymbol{x}) \\
q k_{1, i}(\boldsymbol{x}) \\
\vdots \\
q k_{N, i}(\boldsymbol{x})
\end{array}\right], \quad k=1,2,3, \quad i=0,1,2 \\
\left\{\begin{array}{l}
q k_{0, i}(\boldsymbol{x})=0, \quad 0<i \leq 2, \\
q k_{j, i}(\boldsymbol{x})=0, \quad 1 \leq j \leq i
\end{array}, \quad j=1,2,3, \quad i=0,1,2\right.
\end{gathered}
$$

To reduce computational difficulty, $\hat{\boldsymbol{u}}^{i}(i \geq 2)$ is ignored, then:

$$
\hat{U}=\left[\begin{array}{l}
1 \\
\hat{u}
\end{array}\right]
$$

$\chi(t)$ can be organized as follows:

$$
\boldsymbol{\chi}(t)^{T}=\left[\begin{array}{lll}
q_{\cdot, 0}(\boldsymbol{x}) & q_{\cdot, 1}(\boldsymbol{x}) & q_{,, 2}(\boldsymbol{x})
\end{array}\right]\left[\begin{array}{l}
1 \\
\hat{\boldsymbol{u}}
\end{array}\right]=Q(\boldsymbol{x}) \hat{U}
$$

Invoking (45) into (34a), we can get an equation as follows:

$q_{., 1}(\boldsymbol{x})^{T} M\left(q_{., 0}(\boldsymbol{x})-\boldsymbol{\chi}_{d}\right)+\left(q_{., 1}(\boldsymbol{x})^{T} M q_{., 1}(\boldsymbol{x})+\bar{M}\right) \hat{\boldsymbol{u}}=0$ 
where

$$
\bar{M}=\left[\begin{array}{lll}
\lambda_{3} T & & \\
& \lambda_{3} T & \\
& & \lambda_{3} T
\end{array}\right]
$$

According to (46), the optimal control law is:

$$
\begin{aligned}
& \hat{\boldsymbol{u}}^{*}(t+\zeta)=-\left(q_{., 1}(\boldsymbol{x})^{T} M q_{., 1}(\boldsymbol{x})+\bar{M}\right)^{-1} \\
& * q_{., 1}(\boldsymbol{x})^{T} M\left(q_{., 0}(\boldsymbol{x})-\boldsymbol{\chi}_{d}\right) \quad \zeta \in[0, T]
\end{aligned}
$$

According to $(23), \boldsymbol{u}(t)$ is:

$\boldsymbol{u}(t)=-\left(q_{., 1}(\boldsymbol{x})^{T} M q_{., 1}(\boldsymbol{x})+\bar{M}\right)^{-1} q_{., 1}(\boldsymbol{x})^{T} M\left(q_{., 0}(\boldsymbol{x})-\boldsymbol{\xi}_{d}\right)$

Therefore:

$$
\begin{aligned}
\tau= & \boldsymbol{u}+\mu-\tau_{e n v} \\
= & -\left(q_{., 1}(\boldsymbol{x})^{T} M q_{., 1}(\boldsymbol{x})+\bar{M}\right)^{-1} q_{., 1}(\boldsymbol{x})^{T} M\left(q_{., 0}(\boldsymbol{x})-\boldsymbol{\chi}_{d}\right) \\
& +\mu-\tau_{e n v}
\end{aligned}
$$

As is known, the computation burden [26] is an important issue for real-time control in the applications. According to the above process of NMPC controller design, it can be seen that the highest derivative order is only chosen as 3 . Hence, the computation burden is acceptable for the system.

\section{Stability Analysis}

\subsection{Case in Non-Singular Space}

$N_{s}=\left\{x \in R^{n}:\left|L_{g_{1}} L_{f}^{r-2} h_{1}(x)\right|>\sigma\right\}$ is defined as a closure of non-singular space [27], $r$ represents the relative degree of nonlinear system and $\sigma$ is the radius of $N_{s}$. First, we define that:

$$
\left\{\begin{array}{l}
z_{1}=h_{1}(x)-y_{1 d} \\
z_{2}=L_{f} h_{1}(x)-\dot{y}_{1 d} \\
\vdots \\
z_{r-1}=L_{f}^{r-2} h_{1}(x)-y_{1 d}^{(r-2)}
\end{array}\right.
$$

Then, the part of state equations (16) in which only $h_{1}(x)$ is considered is given as follows:

$$
\begin{aligned}
& \dot{\boldsymbol{x}}=f(\boldsymbol{x})+g(\boldsymbol{x}) \boldsymbol{u} \\
& \boldsymbol{y}_{1}=h_{1}(\boldsymbol{x})
\end{aligned}
$$

For simplicity, (51) is called the subsystem. It can be rewritten as follows:

$$
\left\{\begin{array}{l}
\dot{z}_{1}=z_{2} \\
\vdots \\
\dot{z}_{r-2}=z_{r-1} \\
\dot{z}_{r-1}=L_{f}^{r-1} h_{1}(x)-y_{1 d}^{(r-1)}+L_{g_{1}} L_{f}^{r-2} h_{1}(x) u_{1}
\end{array}\right.
$$

Theorem 1 can be obtained as follows.

Theorem 1. If the subsystem (52) under the action of $u_{1}(t)$ satisfies the following assumption:

$$
\frac{\lambda_{3} T}{\sigma^{2}} \ll M_{r, r}^{(1)}, \frac{T^{r-2}}{\sigma^{2}} \ll M_{r, r}^{(1)}
$$

The subsystem can be regarded as a linear system whose system matrix is written as follows:

$$
A_{1}=\left[\begin{array}{ccccc}
0 & 1 & 0 & \cdots & 0 \\
0 & 0 & 1 & \cdots & 0 \\
\vdots & \vdots & \vdots & \ddots & \vdots \\
0 & 0 & 0 & \cdots & 1 \\
-\frac{M_{r, 1}^{(1)}}{M_{r, r}^{(1)}} & -\frac{M_{r, 2}^{(1)}}{M_{r, r}^{(1)}} & -\frac{M_{r, 3}^{(1)}}{M_{r, r}^{(1)}} & \cdots & -\frac{M_{r, r-1}^{(1)}}{M_{r, r}^{(1)}}
\end{array}\right]
$$

According to the stability criterion of the linear system, the necessary condition for the stabilization of the subsystem (52) is:

$$
\max \left\{\operatorname{Re} \lambda\left(A_{1}\right)\right\}<0
$$

Proof: Assuming $x=\left[\begin{array}{lll}f(t) & \cdots & f^{(n)}(t)\end{array}\right]^{T}$, we can get that $\boldsymbol{\xi}_{1}^{T} x=f(t+\zeta)-o\left(\zeta^{n}\right)$ by using Taylor's formula, $\boldsymbol{\xi}_{1}$ is the element of $\boldsymbol{\xi}(\zeta)$.

Apparently, the latter element is a derivative of the former element with respect to the time in vector $x$. If $x=\left[x_{1}, *, x_{2}\right]^{T}$, then:

$$
\begin{array}{r}
\boldsymbol{\xi}_{1}^{T}\left[\begin{array}{l}
x_{1} \\
* \\
0
\end{array}\right]=f(t+\zeta)-o\left(\zeta^{m}\right) \\
\boldsymbol{\xi}_{1}^{T}\left[\begin{array}{l}
0 \\
* \\
x_{2}
\end{array}\right]=\boldsymbol{\xi}_{1}^{T}\left[\begin{array}{l}
0 \\
* \\
0
\end{array}\right]+\left(\boldsymbol{\xi}_{1}^{T} x-\boldsymbol{\xi}_{1}^{T}\left[\begin{array}{l}
x_{1} \\
* \\
0
\end{array}\right]\right) \\
=\boldsymbol{\xi}_{1}^{T}\left[\begin{array}{l}
0 \\
* \\
0
\end{array}\right]+o\left(\zeta^{m}\right)-o\left(\zeta^{n}\right)=\frac{\zeta^{m}}{m !} \times *+o\left(\zeta^{m}\right)
\end{array}
$$


When $x_{2}$ and determining element $*$ does not meet the derivative relationship as above, $x_{2}$ can be broken down. If the derivative of $*$ is easy to obtain, (57) will be always met. Therefore, $x_{2}$ could have more freedom forms.

When $x \in N_{s}$, according to $q 1_{., 0}(x), q 1_{., 1}(x)$ and (57), we can obtain that:

$$
\boldsymbol{\xi}_{1}^{T} q 1_{., 1}(\boldsymbol{x})=L_{g_{1}} L_{f}^{r-2} h_{1}(x) \frac{\zeta^{r-1}}{(r-1) !}+o\left(\zeta^{r-1}\right)
$$

Further,

$$
\begin{aligned}
q 1_{., 1}(x)^{T} M^{(1)}= & \left.\lambda_{1} q 1_{., 1}(x)^{T} \boldsymbol{\xi}_{1} \boldsymbol{\xi}_{1}^{T}\right|_{\zeta=T} \\
& +\int_{0}^{T} \lambda_{2} q 1_{., 1}(x)^{T} \boldsymbol{\xi}_{1} \boldsymbol{\xi}_{1}^{T} d \zeta \\
= & L_{g_{1}} L_{f}^{r-2} h_{1}(x)\left(\left.\lambda_{1} \frac{\zeta^{r-1}}{(r-1) !} \boldsymbol{\xi}_{1}^{T}\right|_{\zeta=T}\right. \\
& \left.+\int_{0}^{T} \lambda_{2} \frac{\zeta^{r-1}}{(r-1) !} \boldsymbol{\xi}_{1}^{T} d \zeta\right) \lambda_{1} o\left(T^{r-1}\right) \\
& +\lambda_{2} o\left(T^{r-2}\right) \\
= & L_{g_{1}} L_{f}^{r-2} h_{1}(x) M_{r, .}^{(1)}(x)+o\left(T^{r-2}\right)
\end{aligned}
$$

Similarly,

$$
\begin{aligned}
& q 1_{., 1}(\boldsymbol{x})^{T} M^{(1)} q 1_{., 1}(\boldsymbol{x})=M_{r, r}^{(1)}(\boldsymbol{x})\left(L_{g_{1}} L_{f}^{r-2} h_{1}(x)\right)^{2}+o\left(T^{r-2}\right) \\
& q 1_{., 1}(\boldsymbol{x})^{T} M^{(1)}\left(q 1_{., 0}(\boldsymbol{x})-\chi_{1 d}\right) \\
& =L_{g_{1}} L_{f}^{r-2} h_{1}(x)\left(\sum_{i=1}^{r-1} M_{r, i}^{(1)} z_{i}+M_{r, r}^{(1)}\left(L_{f}^{r-1} h_{1}(x)-y_{1 d}^{(r-1)}\right)\right) \\
& \quad+o\left(T^{r-2}\right)
\end{aligned}
$$

Therefore:

$\boldsymbol{u}_{1}(t)=-\frac{\sum_{i=1}^{r-1} M_{r, i}^{(1)} z_{i}+M_{r, r}^{(1)}\left[L_{f}^{r-1} h_{1}(x)-y_{1 d}^{(r-1)}\right]+\frac{o\left(T^{r-2}\right)}{\left(L_{g_{1}} L_{f}^{r-2} h_{1}(x)\right)^{2}}}{M_{r, r}^{(1)}+\frac{\lambda_{3} T}{\left(L_{g_{1}} L_{f}^{r-2} h_{1}(x)\right)^{2}}+\frac{o\left(T^{r-2}\right)}{\left(L_{g_{1}} L_{f}^{r-2} h_{1}(x)\right)^{2}}}$

If the assumption (53) is satisfied, $\boldsymbol{u}_{1}(t)$ can be approximated as follows:

$$
\boldsymbol{u}_{1}(t) \approx-\sum_{i=1}^{r-1} \frac{M_{r, i}^{(1)}}{M_{r, r}^{(1)}} z_{i}+L_{f}^{r-1} h_{1}(x)-y_{1 d}^{(r-1)}
$$

Invoking (63) into (51), Theorem 1 can be proved.

\subsection{Case in Singular Space}

Here, $L_{g_{1}} L_{f}^{r-2} h_{1}(x) \approx 0, L_{g_{1}} L_{f}^{r-1} h_{1}(x) \in o(1)$. We just need to add $z_{r}=L_{f}^{r-1} h_{1}(x)-y_{1 d}^{(r-1)}$ into (50), the new state equation of the subsystem are as follows:

$$
\left\{\begin{array}{l}
\dot{z}_{1}=z_{2} \\
\vdots \\
\dot{z}_{r-1}=z_{r} \\
\dot{z}_{r}=L_{f}^{r} h_{1}(x)-y_{1 d}^{(r)}+L_{g_{1}} L_{f}^{r-1} h_{1}(x) u_{1}
\end{array}\right.
$$

Similarly, Theorem 2 can be obtained as follows.

Theorem 2. If the subsystem (64) under the action of $\boldsymbol{u}_{1}(t)$ satisfies the following assumption:

$$
\begin{aligned}
\frac{\lambda_{3} T}{\left(L_{g_{1}} L_{f}^{r-1} h_{1}(x)\right)^{2}} & \ll M_{r+1, r+1}^{(1)}, \frac{T^{r-1}}{\left(L_{g_{1}} L_{f}^{r-1} h_{1}(x)\right)^{2}} \\
& \ll M_{r+1, r+1}^{(1)}
\end{aligned}
$$

The subsystem can also be approximated as a linear system whose system matrix is given as follows:

$$
A_{2}=\left[\begin{array}{ccccc}
0 & 1 & 0 & \cdots & 0 \\
0 & 0 & 1 & \cdots & 0 \\
\vdots & \vdots & \vdots & \ddots & \vdots \\
0 & 0 & 0 & \cdots & 1 \\
-\frac{M_{r+1,1}^{(1)}}{M_{r+1, r+1}^{(1)}} & -\frac{M_{r+1,2}^{(1)}}{M_{r+1, r+1}^{(1)}}-\frac{M_{r+1,3}^{(1)}}{M_{r+1, r+1}^{(1)}} & \cdots & -\frac{M_{r+1, r}^{(1)}}{M_{r+1, r+1}^{(1)}}
\end{array}\right]
$$

According to the stability criterion of the linear system, the necessary condition for the stabilization of the subsystem (64) is as follows:

$$
\max \left\{\operatorname{Re} \lambda\left(A_{2}\right)\right\}<0
$$

Proof: The proof of Theorem 2 is similar to Theorem 1 .

\subsection{Selection of Parameters}

According to the analysis, the closed-loop system will be stable if the conditions (53), (55), (65) and (67) can be met under the action of $u_{1}$. Because $A_{1}$ and $A_{2}$ are determined by $M^{(1)}, T, \lambda_{1}$ and $\lambda_{2}$ should be chosen suitably. According to the literature [17], $T$ plays a major role compared with $\lambda_{1} / \lambda_{2}$. Therefore, the value of $T$ influences the performance of system strongly. In addition, to meet (53) and (65), $\lambda_{3}$ can be set to zero.

The control laws $u_{1}, u_{2}$ and $u_{3}$ are independent, so we can use the same method to analyse the stability of $u_{2}$ and $u_{3}$. We can find that $\lambda_{1}, \lambda_{2}, \lambda_{3}$ and $T$ are occupied in $u_{1}, u_{2}$ and $u_{3}$, so there is no need to choose them again.

\section{Simulation}

One dynamic positioning ship [28] is used as case study. The main particulars of ship, hydrodynamic coefficients of ship and constraints on the thrusters' generalized forces are given in Tables 1-3:

According to the previous analysis, $\lambda_{1}, \lambda_{2}$ and $\lambda_{3}$ can be set as 1.0, 0.0005 and 0, respectively. However, the predictive period $T$ plays a major role in the system compared with $\lambda_{1}, \lambda_{2}$ and $\lambda_{3}$. To find the ideal predictive period, the simulation experiments with different predictive periods were carried out as follows. Initial position and heading angle are $\left(0 \mathrm{~m}, 0 \mathrm{~m}, 0^{\circ}\right)$, desired position and heading angle are $\left(100 \mathrm{~m}, 100 \mathrm{~m}, 30^{\circ}\right)$. Wind velocity 
Table 1

Main Particulars of DP Ship

\begin{tabular}{|l|l|}
\hline Length $(\mathrm{LOA})$ & $76.2 \mathrm{~m}$ \\
\hline Beam $(B)$ & $18.8 \mathrm{~m}$ \\
\hline Draught $(T)$ & $6.25 \mathrm{~m}$ \\
\hline Displaced volume $(\nabla)$ & $4200 \mathrm{~m}^{3}$ \\
\hline
\end{tabular}

Table 2

Hydrodynamic Coefficients

\begin{tabular}{|l|c|c|}
\hline $\begin{array}{l}\text { Hydrodynamic } \\
\text { coefficients }\end{array}$ & Value & Unit \\
\hline$X_{u}$ & $-5.3122 \times 10^{6}$ & $\mathrm{~kg} \cdot \mathrm{m}^{-1}$ \\
\hline$Y_{v}$ & $-2.7229 \times 10^{5}$ & $\mathrm{~kg} \cdot \mathrm{m}^{-1}$ \\
\hline$Y_{r}$ & $4.3933 \times 10^{6}$ & $\mathrm{~kg}$ \\
\hline$N_{v}$ & $4.3933 \times 10^{6}$ & $\mathrm{~kg}$ \\
\hline$N_{r}$ & $-4.1894 \times 10^{8}$ & $\mathrm{~kg} \cdot \mathrm{m}$ \\
\hline
\end{tabular}

Table 3

Constraints on the Thrusters' Generalized Forces

\begin{tabular}{|l|c|}
\hline Maximal pitch thrust & $1000 \mathrm{kN}$ \\
\hline Maximal roll thrust & $300 \mathrm{kN}$ \\
\hline Maximal yaw moment & $7620 \mathrm{kN} \mathrm{m}$ \\
\hline
\end{tabular}
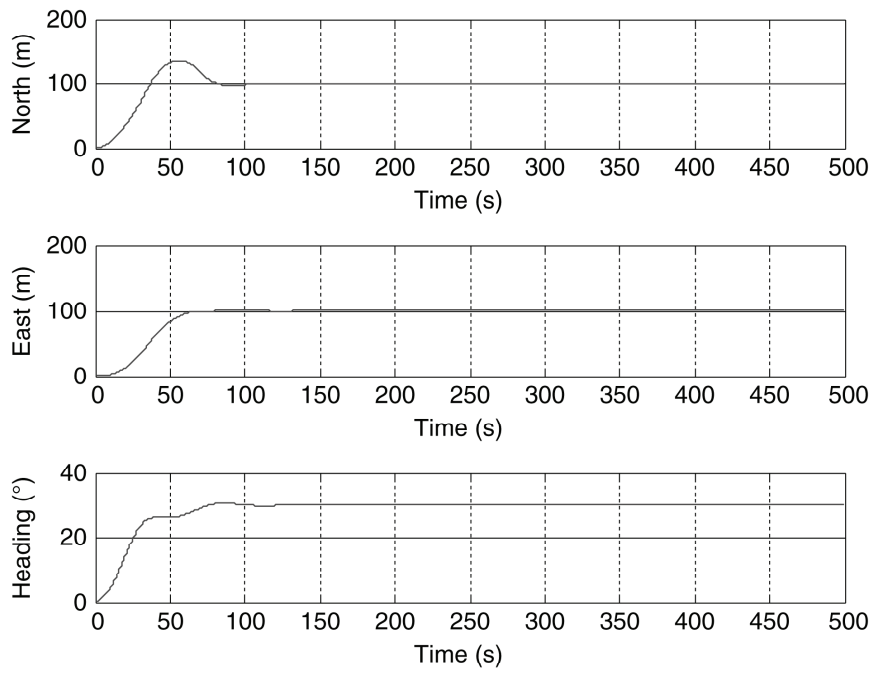

Figure 2. Positions and Euler angle of unified model $(T=7.5 \mathrm{~s})$.

and direction are $\left(10 \mathrm{~m} / \mathrm{s}, 90^{\circ}\right)$, significant wave height and wave direction are $\left(3 \mathrm{~m}, 100^{\circ}\right)$, current velocity and direction are $\left(0.5 \mathrm{~m} / \mathrm{s}, 120^{\circ}\right)$. The position and heading angle of unified model with different predictive periods are shown in Figs. 2-4:

From Figs. 2 to 4 , it can be seen that the controller can make the DP vessel move to the target position and heading
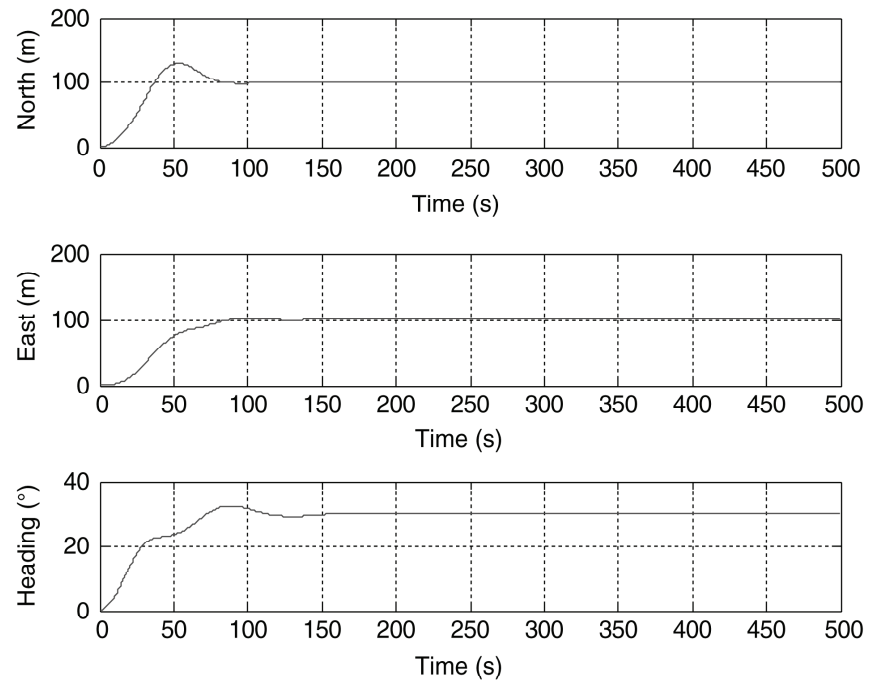

Figure 3. Positions and Euler angle of unified model $(T=9.0 \mathrm{~s})$.
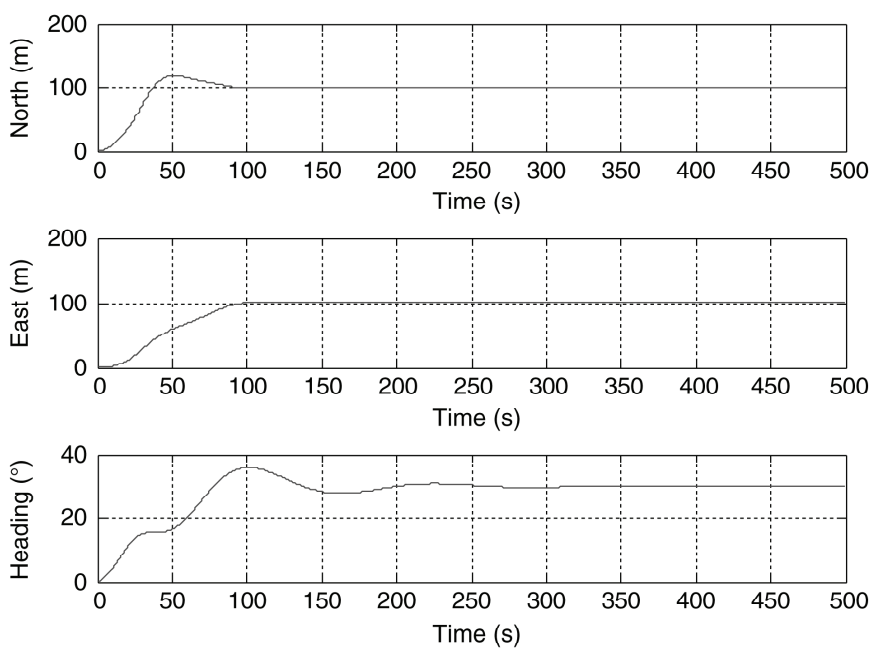

Figure 4. Positions and Euler angle of unified model $(T=11.0 \mathrm{~s})$.

with different predictive periods. However, the response time becomes longer with the increase in the predictive period. Meanwhile, the overshoot along the north becomes obviously smaller with the increase in the predictive period. Therefore, the predictive period $T$ can be chosen as $9.0 \mathrm{~s}$ based on the above analysis.

To verify the performance of the designed controllers, the simulation experiments should be carried out under different environmental conditions. For different environmental conditions, environment condition 1 is as similar as the simulation condition for the selection of predictive period; environment condition 2 is defined as follows: wind velocity and direction are $\left(5 \mathrm{~m} / \mathrm{s}, 90^{\circ}\right)$, significant wave height and wave direction are $\left(1 \mathrm{~m}, 100^{\circ}\right)$, current velocity and direction are $\left(0.1 \mathrm{~m} / \mathrm{s}, 120^{\circ}\right)$. Obviously, environment condition 1 is harsher than condition 2 . The simulation results under different environment conditions are shown in Figs. 5-8: 

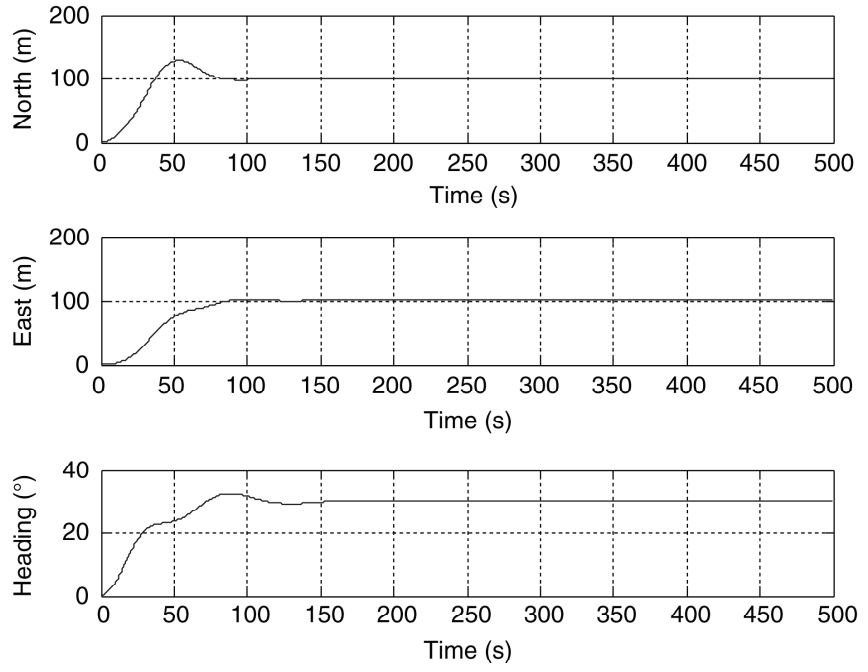

Figure 5. Positions and Euler angle of DP ship under environment condition 1.
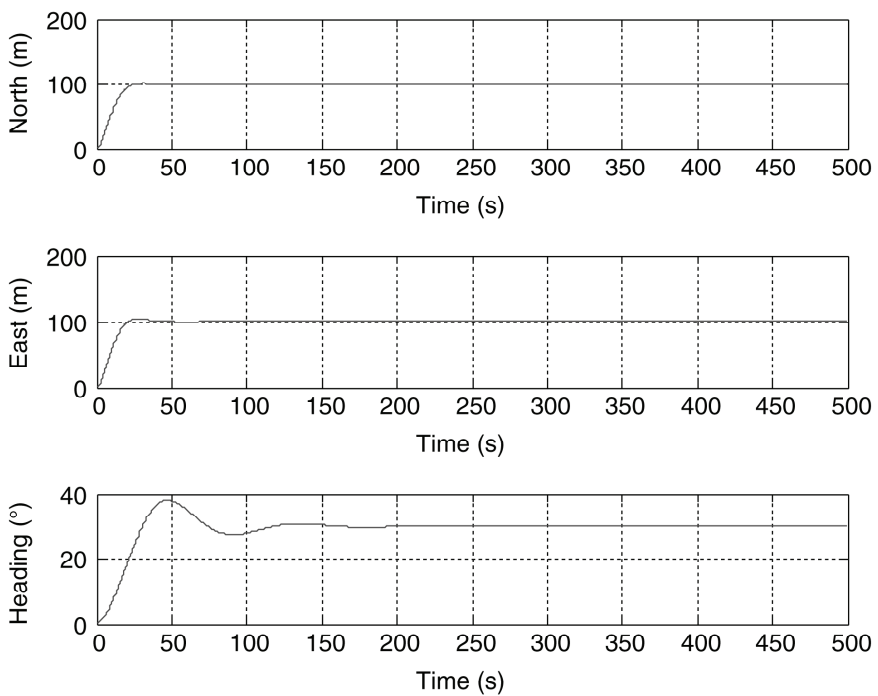

Figure 6. Positions and Euler angle of DP ship under environment condition 2 .

From Figs. 5 to 6 , the DP vessel can both move to the target position and heading under the action of the proposed controller. However, the response time becomes obviously longer and the overshoot along the north becomes obviously bigger under environmental condition 1 due to the harsher sea state and larger generalized thrusts. Although the highest derivative order is simplified to 3 only for the reduction of computation burden, the positioning effect can still meet the positioning requirement.

From Figs. 7 to 8, it can be seen that the thrusts and moment of the thrusters under environmental condition 1 are obviously larger than those under environmental condition 2. But they are all within the constraints in Table 2. From Fig. 7, it can be seen that the thrusts and moment under environmental condition 1 have reached to the constraints, so the northern desired position is reached
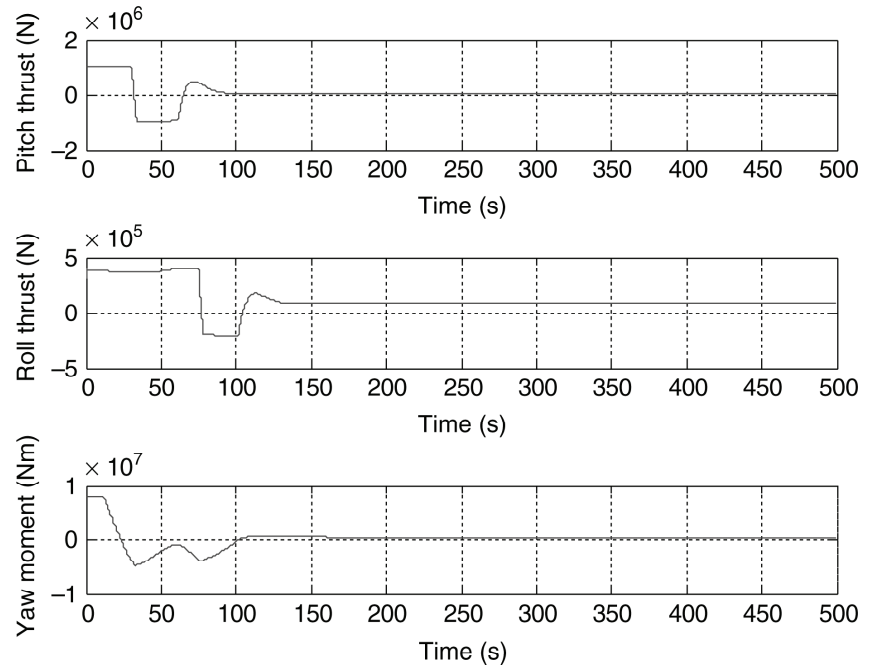

Figure 7. Forces and moment of thrusters under environment condition 1 .
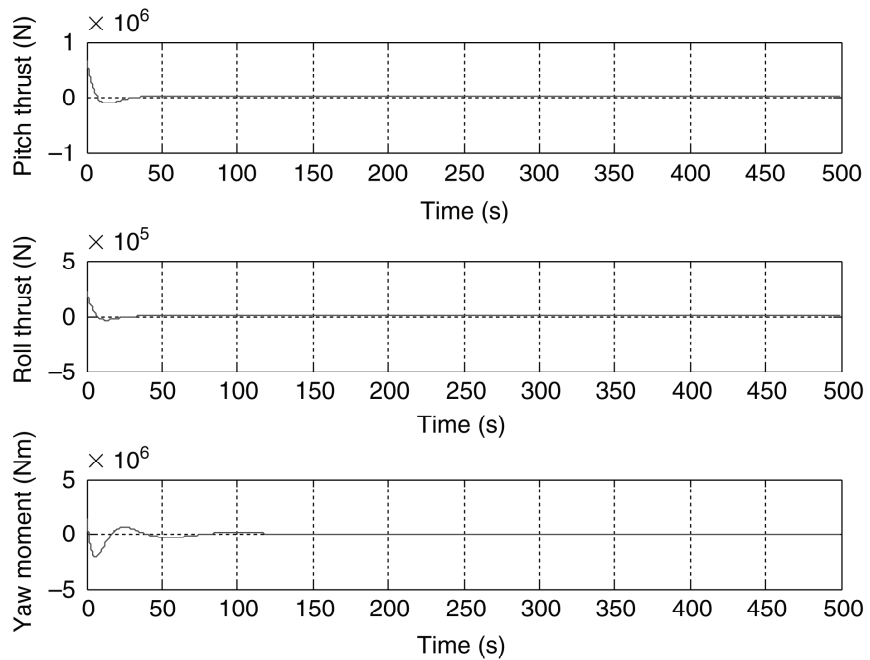

Figure 8 . Forces and moment of thrusters under environment condition 2 .

more quickly than the eastern desired position due to the larger constraints of pitch thrust.

Moreover, it is known that the DP vessel system is a system with ill-defined relative degree from the process of controller design. However, the outputs of the NMPC controller are still continuous and smooth. So, it is proved that the improved NMPC controller is non-switched and able to avoid the chattering phenomenon. Moreover, this algorithm is suitable for the more general nonlinear systems with ill-defined relative degree.

\section{Conclusion}

Unified modelling and NMPC design for DP of deep-sea vessels are investigated in this paper. The unified mathematical model of the DP ship is derived in which the memory effect of the fluid is included. An improved analytic 
NMPC method for nonlinear systems with ill-defined relative degree is applied in the unified model of the DP vessel. The controller is successfully demonstrated by simulation examples. By choosing an appropriate predictive period, the DP ship can move to the desired position and heading quickly and accurately under different environmental conditions. However, parameters' selection of the controllers is complex and difficult, where the simulation results are not perfect but acceptable for practical applications. Intelligence algorithms such as neural networks or genetic algorithm could be considered for parameters optimization in the following work. In addition, time delay and uncertainty are so prevalent in practical systems that they should be considered in the future work. Robust sliding mode control methods will be effective to deal with the delay and uncertainty of the systems.

\section{Acknowledgements}

This work was supported by the National Natural Science Foundation of China (No. 51209056) and Fundamental Research Funds for the Central Universities (No. HEUCFX41501).

\section{Conflict of Interests}

The authors declare that there is no conflict of interests regarding the publication of this paper.

\section{References}

[1] T.I. Fossen, Mathematical models of ships and underwater vehicles, J. Baillieul and T. Samad, (eds.), Encyclopedia of Systems and Control, Springer, 2014.

[2] A.J. Sørensen, A survey of dynamic positioning control systems, Annual Reviews in Control, 35(1), 2011, 123-136.

[3] T.I. Fossen, Handbook of marine craft hydrodynamics and motion control (Chichester: Wiley, 2011).

[4] T.I. Fossen, A nonlinear unified state-space model for ship maneuvering and control in a seaway, International Journal of Bifurcation and Chaos, 15(09), 2005, 2717-2746.

[5] A.J. Sørensen, Dynamic positioning control systems for ships and underwater vehicles, Encyclopedia of Systems and Control, 2014.

[6] H. Fay, Dynamic positioning systems, principles, design and applications (Paris, France: Editions Technip, 1989).

[7] A.J. Sørensen, F. Dukan M. Ludvigsen, D.A. Fernandez, Development of dynamic positioning and tracking system for the ROV Minerva, Further advances in unmanned marine vehicles, IET Digital Library, 2012, 113-128.

[8] S. Islam, X.P. Liu, and A.E. Saddik, Adaptive sliding mode control of unmanned four rotor flying vehicle, International Journal of Robotics 85 Automation, 30(2), 2015, 140-148.

[9] J.K. Rai, R.P. Tewari, and A.D. Chandra, An optimal control of biped robot for human-like walking, International Journal of Robotics \& Automation, 28(2), 2013, 129-136.

[10] C.J. Zhang, H.P. Hu, and Y.Q. Zhang, Intelligent control of time-delayed system, Applied Mechanics \&6 Materials, 721, 2015, 253-256.

[11] E.F. Camacho and C. Bordons, Distributed model predictive control, Optimal Control Applications and Methods, 34(2), 2013, 503.

[12] R. Huang, V.M. Zavala, and L.T. Biegler, Advanced step nonlinear model predictive control for air separation units, Journal of Process Control, 19(4), 2009, 678-685.
[13] J. Richalet, A. Rault, and J.L. Testud, Model predictive heuristic control: Applications to industrial processes, Automatica, 14(5), 1978, 413-428.

[14] T. Fan and C.W.D. Silva, Dynamic modelling and model predictive control of flexible-link manipulators, International Journal of Robotics 83 Automation, 23(4), 2008, 227-234.

[15] S. Di Cairano, M. Brand, and S.A. Bortoff, Projection-free parallel quadratic programming for linear model predictive control, International Journal of Control, 86(8), 2013, 13671385.

[16] L.C. Kwek, A.W.C. Tan, and E.K. Wong, Generalized predictive control on a robotic manipulator system, International Journal of Robotics 85 Automation, 28(3), 2013, 277-283.

[17] W.H. Chen, D.J. Ballance, and P.J. Gawthrop, Optimal control of nonlinear systems: A predictive control approach, Automatica, 39(4), 2003, 633-641.

[18] W.H. Chen, Predictive control of general nonlinear systems using approximation, Control Theory and Applications, IEE Proceedings, 151(2), 2004, 137-144.

[19] X. Peng, S. Jia, and X. Wang, Analytic model predictive control of uncertain nonlinear systems: A fuzzy adaptive approach, Mathematical Problems in Engineering, 2015, 1-9.

[20] G.Y. Zhang, Z. Yang, and H.Z. Tan, Research on non-switch analytic nonlinear model predictive control method for a class of nonlinear systems, Acta Automatica Sinica, 34(34), 2008, $1147-1156$.

[21] W.H. Chen and D.J. Ballance, On a switching control scheme for nonlinear systems with ill-defined relative degree, Systems 83 Control Letters, 47(2), 2002, 159-166.

[22] O. Faltinsen and T. Svensen, Incorporation of seakeeping theories in CAD, Ships, 1990.

[23] T. Perez, TI. Fossen, and A. Srensen, A discussion about sea keeping and manoeuvering models for surface vessels, NTNU, 2004.

[24] W.H. Chen, Predictive control of general nonlinear systems using approximation, Control Theory and Applications, IEE Proceedings, 151(2), 2004, 137-144.

[25] F.P. Miller, A.F. Vandome, J. Mcbrewster, et al. Lie derivative, Communications 83 Control Engineering, 2010, 307-330.

[26] Q.Q. Jia, R.B. Wu, Detection of fast air moving din targets via STAP with low computation burden, Radar Conference 2013, IET International, IET, Xi'an, 2013, 1-5.

[27] Z. Yang, L. Peng, and Z. Fan, Suboptimal control study for a class of nonlinear systems, Control and Decision, 24(12), 2009, 1835-1836.

[28] Y Wang, Y Sui, and WU Jing, Marine dynamic position system based on nonlinear model predictive control, Journal of Harbin Engineering University, 34(1), 2013, 110-115.

\section{Biographies}

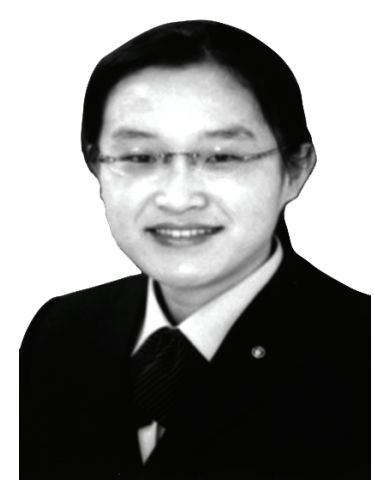

Yuanhui Wang was born in 1981, doctor of engineering. She is a Ph.D. associate supervisor and an associate professor at College of Automation, Harbin Engineering University. She finished her B.S. degree, M.S. degree and Ph.D. degree in Harbin Engineering University. After finishing her Ph.D. degree, now she is working in College of Automation in Harbin Engineering University. Her research interests include ship motion and control, intelligent control, hybrid control, dynamic positioning system for ships, hovercraft safe navigation, coordinated formation and collision avoidance control for multiple ships. 


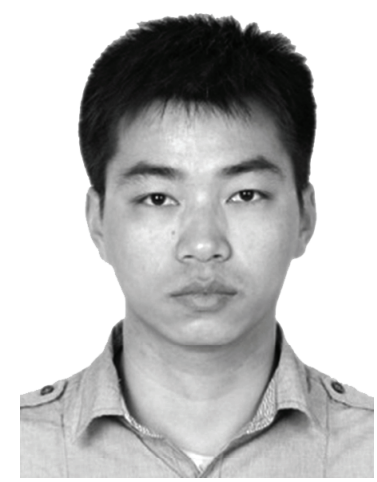

Yulong Tuo received a B.S. degree in College of Automation from Harbin Engineering University, Harbin, China, in 2013. Now, he is currently working toward the Ph.D. degree in the College of Automation, Harbin Engineering University. His current research interests include advanced mathematical modelling for vessels, dynamic positioning control, intelligent control, trajectory tracking and collision avoidance.

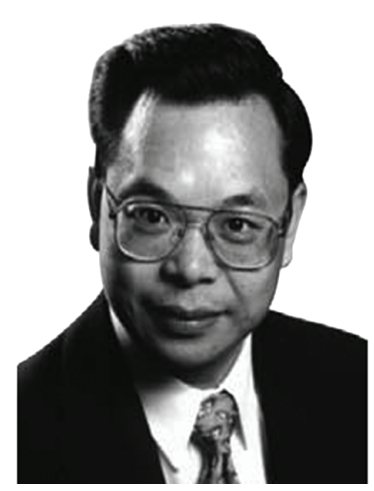

Simon $X$. Yang received the B.Sc. degree in Engineering Physics from Beijing University, Beijing, China, in 1987, the first of two M.Sc. degrees in Biophysics from the Chinese Academy of Sciences, Beijing, in 1990, the second M.Sc. degree in Electrical Engineering from the University of Houston, Houston, TX, in 1996, and the Ph.D. degree in Electrical and Computer Engineering from the University of Alberta, Edmonton, AB, Canada, in 1999. Dr. Yang is currently Professor and Head of the Advanced Robotics and Intelligent Systems Laboratory at the University of Guelph, Guelph, ON, Canada. His research interests include intelligent systems, robotics, sensors and multi-sensor fusion, wireless sensor networks, control systems, and computational neuroscience. Prof. Yang has been very active in professional activities. He serves as Editor-in-Chief of International Journal of Robotics and Automation, Journal of Robotics and Artificial Intelligence, and International Journal of Complex Systems - Computing, Sensing and Control; an Advisory Editorial Board member of Intelligent Automation and Soft Computing; and Associate Editor of IEEE Transactions on Cybernetics, and several other journals. He was the General Chair of the 2011 IEEE International Conference on Logistics and Automation, and the Program Chair of the 2015 IEEE International Conference on Information and Automation. Among many of his awards, he was a recipient of the Distinguished Professor Award at the University of Guelph.

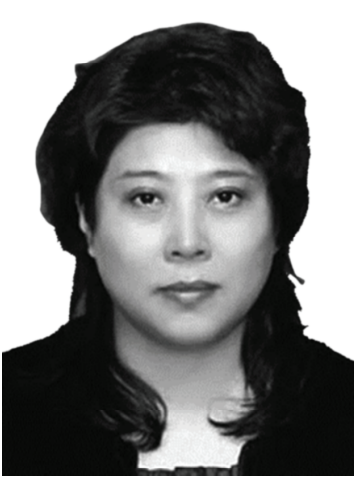

Mingyu Fu was born in 1964. She finished her B.S., M.S., in Harbin marine engineering college, and Ph.D. degree in Harbin Engineering University. She began to work in the School of Control Science and Engineering of Harbin Engineering University after she got the master's degree. Now, she is a professor and a Ph.D. supervisor of Control Science and Engineering at College of Automation, Harbin Engineering University. Her research interests include ship motion and control, multi-sensor fusion, intelligent system control. The main research directions include dynamic positioning system for ships, hovercraft safe navigation and coordinated formation control for multiple ships. 